\title{
LA CONSTRUCCIÓN DE LOS EDIFICIOS ESCOLARES: CONFLUENCIA DE LOS DISCURSOS PEDAGÓGICOS Y POLÍTICOS A FINES DEL SIGLO XIX
}

\author{
Francisco Hernández Ortiz \\ Benemérita y Centenaria Escuela Normal del Estado de San Luis Potosí México \\ fhernandez@beceneslp.edu.mx
}

\begin{abstract}
RESUMEN
Este trabajo da a conocer las condiciones en las que surgen los primeros edificios escolares a fines del siglo XIX en las ciudades de Buenos Aires, Argentina y San Luis Potosí, México respectivamente. La metodología, analítico- sintético, ayudó a la reconstrucción histórica, permitió identificar rasgos comunes y diferenciados en el origen de los primeros edificios escolares en las dos ciudades latinoamericanas. Los conceptos bajo los cuales se hace la argumentación son: pedagogía, educación moderna, higiene escolar, modernidad, patrimonio histórico educativo y Estado. El estudio muestra el paralelismo del surgimiento de los primeros edificios escolares, espacios para la educación escolarizada bajo la tutela del Estado, con una infraestructura específica, como resultado de los avances de la pedagogía, la medicina, la arquitectura e ingeniería, como evidencia de la modernidad de fines del siglo XIX en estas dos regiones del continente americano.
\end{abstract}

Palabras clave: Higiene. Modernización. Pedagogía. Educación. Espacio. Edificio escolar. Patrimonio histórico educativo.

\section{A CONSTRUÇÃO DE EDIFÍCIOS ESCOLARES: CONFLUÊNCIA DE DISCURSOS PEDAGÓGICOS E POLÍTICOS NO FIM DO SÉCULO XIX}

\section{RESUMO}

Este trabalho revela as condições em que os primeiros prédios escolares surgiram no final do século XIX nas cidades de Buenos Aires, Argentina e San Luis Potosí, México, respectivamente. A metodologia analítico-sintética contribuiu para a reconstrução histórica, permitindo identificar características comuns e diferenciadas na origem dos primeiros prédios escolares das duas cidades latino-americanas. Os conceitos sob os quais o argumento é apresentado são: pedagogia, educação moderna, higiene escolar, modernidade, patrimônio educacional histórico e Estado. $\mathrm{O}$ estudo mostra o paralelismo do surgimento dos primeiros prédios escolares, espaços de educação escolar sob a tutela do Estado, com infraestrutura específica, como resultado dos avanços em pedagogia, medicina, arquitetura e engenharia, como evidência da Modernidade do final do século XIX nessas duas regiões do continente americano.

Palavras-chave: Higiene. Modernização. Pedagogia. Educação. Espaço. Construção de escola. Patrimônio histórico educacional. 


\title{
THE CONSTRUCTION OF SCHOOL BUILDINGS: CONFLUENCE OF PEDAGOGICAL AND POLITICAL DISCOURSES AT THE END OF THE 19TH CENTURY
}

\begin{abstract}
This work reveals the conditions in which the first school buildings emerged at the end of the 19th century in the cities of Buenos Aires, Argentina and San Luis Potosí, Mexico respectively. The methodology, analytical-synthetic, helped to the historical allowed to identify common and differentiated features in the origin of the first school buildings in the two Latin American cities. The concepts under which the argument is made are: pedagogy, modern education, school hygiene, modernity, historical educational heritage and State. The study shows the parallelism of the emergence of the first school buildings, spaces for school education under the tutelage of the State, with a specific infrastructure, as a result of advances in pedagogy, medicine, architecture and engineering, as evidence of the Modernity of the late nineteenth century in these two regions of the Americas.
\end{abstract}

Keywords: Hygiene. Modernization. Pedagogy. Education. Space. School building. Educational historical heritage.

\section{LA CONSTRUCTION DES BÂTIMENTS SCOLAIRES: CONFLUENCE DES DISCOURS PÉDAGOGIQUES ET POLITIQUES À LA FIN DU XIXe SIÈCLE}

\section{RÉSUMÉ}

Ce travail révèle les conditions dans lesquelles les premiers bâtiments scolaires sont apparus à la fin du XIXe siècle dans les villes de Buenos Aires, Argentine et San Luis Potosí, Mexique respectivement. La méthodologie, analytique-synthétique, a aidé à la reconstruction historique complétée, a permis d'identifier des caractéristiques communes et différenciées à l'origine des premiers bâtiments scolaires dans les deux villes d'Amérique latine. Les concepts sous lesquels l'argument est avancé sont: la pédagogie, l'éducation moderne, l'hygiène scolaire, la modernité, le patrimoine éducatif historique et l'État. L'étude montre le parallélisme de l'émergence des premiers bâtiments scolaires, des espaces pour l'enseignement scolaire sous la tutelle de l'État, avec une infrastructure spécifique, grâce aux progrès de la pédagogie, de la médecine, de l'architecture et de l'ingénierie, comme preuve de la Modernité de la fin du XIXe siècle dans ces deux régions du continent américain.

Mots-clés: hygiène. Modernisation. Pédagogie. Éducation. Espace. Bâtiment Scolaire. Patrimoine Historique éducatif.

\section{INTRODUCCIÓN}

Este estudio tiene como objeto de investigación: los edificios escolares a fines del siglo XIX, como espacios simbólicos de la modernidad. En ellos se difunde la cultura, la ciencia y la 


\section{$(c)) \mathrm{BY}$}

ideología del Estado. Los referentes geográficos son dos ciudades latinoamericanas: San Luis Potosí, México, y Buenos Aires, Argentina. La construcción de los primeros edificios escolares en estas dos latitudes, permite conocer las causas políticas, educativas y pedagógicas del Estado por transformar la educación pública, de acuerdo a los mandatos constitucionales en cada uno de estos dos países,

La historia de la humanidad, está relacionada con el tiempo y el espacio, en especial con el creado para la convivencia, donde se realizan las actividades de la vida cotidiana. El lugar donde se consumen los alimentos o donde se descansa o se duerme, etc. Así surge el espacio escolar como un sitio a donde se acude para que unos enseñen y otros aprendan. La escuela es un espacio y lugar sociocultural. El espacio escolar tiene diferencias significativas comparado con cualquier otro espacio en donde interactúan las personas (VIÑAO, 1993) porque es ahí donde los alumnos se apropian de la cultura y de la ideología del Estado.

Las construcciones escolares surgen en Europa, se intensificaron desde la segunda mitad del siglo XIX, sirvieron de modelo arquitectónico para los que se construyeron en el continente americano. Los edificios escolares son espacios en donde se socializa y educa a las personas (VIÑAO, 2008). En América es tardía la construcción de edificios exprofeso para la tarea educativa, en México, Argentina y Colombia los edificios eran casas habitación adaptadas, casi siempre descuidadas (MALDONADO, 1999). Coincide con lo encontrado en los archivos sobre los establecimientos públicos en la ciudad de San Luis Potosí, México, estos funcionaban en casas rentadas o de propiedad del gobierno; los edificios carecían de las áreas pedagógicas para la enseñanza y el aprendizaje (HERNÁNDEZ, 2011).

Los estudios sobre el espacio escolar, deben verse desde lo diacrónico, histórico, didáctico y su utilidad para las tareas escolares (GODÍNEZ, 1991). Sólo de esta forma se puede comprender su trascendencia como áreas para la enseñanza, recreación, y apropiación de la cultura. En el estado de conocimiento sobre la arquitectura escolar, se reconoce la influencia suiza, alemana y francesa en la definición conceptual y arquitectónica de los primeros edificios escolares en España en el siglo XIX (RODRÍGUEZ, 2004); este autor, argumenta que el diálogo profesional entre maestros y arquitectos fue fundamental en la definición de espacios escolares. El movimiento de la educación moderna en Europa, obligó a que estos dos profesionales acordaran crear dentro de la distribución arquitectónica del edificio escolar con superficies como biblioteca, salones, sanitarios, 


\section{$(c c)$ EY}

salas, etc., propias para la enseñanza. El edificio escolar, fue una construcción de los imaginarios de la escuela como espacios iluminados por la ciencia y la belleza arquitectónica.

La escuela moderna, en sus inicios se caracteriza por el hacinamiento de los alumnos en el aula, poca iluminación o ventilación, esto provocaba efectos negativos como el cansancio o problemas de salud en los alumnos. Los discursos pedagógicos de la escuela moderna propugnan por crear espacios exprofeso para la enseñanza y el aprendizaje de acuerdo al concepto de educación integral. La escuela debía tener espacios para el cultivo de prácticas pedagógicas donde se fomenta el juego, el estudio, la convivencia; diferente al espacio de la escuela tradicional (LAHOZ, 2010) centrado en el uniespacio para el aprendizaje.

El movimiento internacional sobre higiene y sanidad a fines del siglo XIX, está relacionado con el rol del Estado benefactor, como sucedió en Europa y América. El movimiento de políticas higienistas y de salud, se intensificaron para contrarrestar la mortalidad de la población provocada por epidemias, en parte debido a las condiciones de pobreza e insalubridad, siendo los niños los más vulnerables a las enfermedades y por ende el incremento de decesos infantiles (GUTIÉRREZ, 2010). Las discusiones en los Congresos de Instrucción Primaria de 1889 y 1891 , quedaron sintetizadas en sus respectivos resolutivos: uniformidad de la educación, laicismo, renovación de los métodos, materiales educativos, la formación de los profesores, y la condición de las escuelas para que los alumnos acudan a ellas a recibir educación en las mejores condiciones que señala la pedagogía. En conclusión "se decidió que la uniformidad en la educación nacional consistiría en la enseñanza obligatoria, gratuita y laica" (BAZANT, 1995, p.24).

En la ciudad de México en 1909, se introdujeron medidas higienistas y pedagógicas, implicó adaptar espacios adecuados para la enseñanza; lo que dio pauta a la construcción de los primeros edificios escolares. Con antelación el tema fue discutido en el Congreso Higienista de 1882. "Las modernas recomendaciones no sólo de la higiene sino también de la pedagogía aconsejaban construir la escuela como un lugar exclusivo, que tuviera asignadas tareas propias y definidas" (CHAOUL, 2012, p.252.). Acompañaron a Justo Sierra Ministro de Instrucción y Bellas Artes: "Ezequiel Chávez y Gregorio Torres Quintero, Manuel Uribe y Troncoso, Eugenio Latapí, Jesús González Ureña y su equipo de médicos, arquitectos e ingenieros se convirtieron en los principales promotores de los cambios en lo que respecta a la higiene escolar" (CHAOUL, 2012, p.255.). La rehabilitación, adecuación o construcción de escuelas en el Distrito Federal, inició en 


\section{$(c c)$ EY}

el Porfiriato, pero se intensificó en el periodo posrevolucionario, como política de Estado a lo largo del siglo XX en México.

Para el caso de Colombia, las ideas higienistas se introdujeron desde 1886 con la creación de la Junta Central de Higiene, aunque por las diferencias políticas en el país, las medidas higienistas se aplicaron hasta las primeras décadas del siglo XX (GUTIÉRREZ, 2010). Mientras que en Argentina fue a partir de 1880, cuando dio inicio una transformación educativa para construir un sistema de representación política unificado y organizado por el Estado, para construir la Nación, eso implicó definir el tipo de educación para la formación del nuevo ciudadano (LIONETTI, 2005). Se efectuaron acciones para transformar la educación, se concatenaron medidas higienistas, como el cuidado del espacio físico y su organización espacial en los edificios públicos, como las escuelas públicas. (BARBIERI, 2016). Con la aparición de la ley 1420 de 1884 en la Argentina, se declara a la educación común, laica gratuita y obligatoria: aunado a lo anterior se integró a los ideales liberales los aportes de la medicina, la eugenesia y la puericultura para mejor educar a los niños y niñas cuidando los espacios exprofeso para la educación (LIONETTI, 2011).

Este trabajo tiene como objetivo, dar a conocer las razones pedagógicas y políticas para la construcción de los primeros edificios escolares a fines del siglo XIX en la ciudad de Buenos Aires, Argentina y en la ciudad de San Luis Potosí, México, dentro del marco de la educación moderna. Se parte de dos supuestos: primero, la edificación de los centros escolares en la ciudad de Buenos Aires, Argentina a fines del siglo XIX tuvo como referente los principios pedagógicos de la educación moderna; impulsados con las decisiones políticas que favorecieron construcciones específicas para la tarea educativa, de acuerdo a ciertos ordenamientos de la higiene escolar lo que dio pauta a la cimentación de los primeros centros escolares. Segundo, la edificación de escuelas exprofeso para la tarea educativa, en la ciudad de San Luis Potosí, México, fue tardía con respecto a lo acontecido en la ciudad de Buenos Aires, Argentina. Las preguntas que guían el análisis son: ¿Cuáles fueron las causas sociopolíticas y pedagógicas para la construcción de los edificios escolares en la ciudad de Buenos Aires y en la Ciudad de San Luis Potosí?, ¿Cómo se tradujeron las ideas pedagógicas de la educación moderna en la educación de la ciudad de Buenos Aires y en San Luis Potosí para crear los edificios escolares?, ¿Qué papel jugaron las comisiones pedagógicas u organizaciones que tomaban las decisiones educativas en cada uno de los países? 


\section{$(c)$ EY}

Derivado de una visita en el 2017 a la Biblioteca Nacional de Maestros en la ciudad de Buenos Aires, Argentina, se localizó y analizó el compendio de planos de los edificios escolares de 1894, del cual se recaban los datos para este trabajo. Y se complementa con datos de Diario de Monitor Común, consultado en su versión digital. Del Archivo Histórico del Sistema Educativo Estatal Regular de San Luis Potosí, México, se consultó el acervo de planos de las primeras escuelas públicas del gobierno del estado de San Luis Potosí, México, 1889.

La metodología, se diseñó bajo el método analítico- sintético, implicó hacer tablas clasificatorias para identificar y recabar datos de cada plano: tipo de edificio, domicilio, número de aulas, salas sanitarias, bodegas, oficina, laboratorio o comedor; los datos hicieron visibles las semejanzas y diferencias de los edificios escolares en las dos latitudes. Con el apoyo de conceptos como pedagogía, educación moderna, higiene escolar, modernidad y Estado; se hizo la reconstrucción histórica del surgimiento de los primeros edificios escolares.

\section{HACIA LA CONSTRUCCIÓN DE LOS PRIMEROS EDIFICIOS ESCOLARES EN ARGENTINA.}

Los discursos pedagógicos de la educación moderna y la higiene escolar influyeron en los gobiernos para crear organismos para administrar la educación, renovar los sistemas educativos, construir el modelo de escuela; esto implicó la definición de arquetipos de edificios escolares. Las alocuciones políticas y pedagógicas de erigir edificios escolares exprofeso, fue porque los alumnos que asistían a las escuelas públicas podrían acceder al saber científico, artístico y humanístico en áreas adecuadas para las actividades escolares, como ya sucedía en Europa.

La construcción de los primeros edificios escolares modernos, en la ciudad de Buenos Aires, Argentina; se erigieron en la segunda mitad del siglo XIX. Una evidencia son los planos que datan de 1894, elaborados por el Consejo Nacional de Educación (CNE) y preservados en la Biblioteca Nacional de Maestros (BNM), de los cuales se extrajeron los datos para este trabajo.

El CNE fue creado el 28 de enero de 1881, por el presidente Julio Argentino Roca; en su inicio tuvo jurisdicción solamente en la ciudad de Buenos Aires, posteriormente fue de cobertura nacional. Este consejo se convirtió en "la voz oficial del gobierno del sistema educativo nacional." (MORENGO, 1991, p.71). Como organismo del Estado, sus atribuciones, fueron "arbitrar las disposiciones concernientes a garantizar la fiel inversión de los fondos que se distribuyen a las 


\section{$(c c)$ EY}

provincias en virtud de la ley de subvenciones a la educación común, y propuso al Ministerio de Instrucción Primaria su adopción.”" También se encargó de todos los expedientes o gestiones sobre construcción y reparación de edificios de escuelas en las provincias, colonias y territorios nacionales, adquisición de mobiliario, material científico y pago de sueldos del personal docente y administrativo, todo a cargo del Tesoro Nacional de Argentina.

El compendio de planos de los primeros edificios escolares de fines del siglo XIX bajo la responsabilidad del CNE, indican el interés político del gobierno argentino, de construir espacios educativos acordes a las exigencias de la pedagogía moderna y de la higiene escolar; cada uno de los planos indica que dichos edificios fueron concebidos como espacios exclusivos para la tarea educativa.

Los edificios escolares son construcciones simbólicas de la modernidad, en ellos se conjugan las aportaciones de tres disciplinas: la arquitectura, la higiene y la pedagogía, en cada uno, subyace el ideal educativo asimilado por los intelectuales y políticos dirigentes del país de fines del siglo XIX. El edificio simboliza el espacio construido para la escuela que se convierte en un "dispositivo de generación de ciudadanos- sostienen algunos liberales-, o de proletarios - según algunos marxistas-, pero no solo eso.” (PINEAU, DUSSEL, CARUSO, 2001, p.8). Es también el espacio de confluencia social, en donde los alumnos se apropian de los contenidos culturales, ahí se construye la identidad y sentido de pertenencia a la nación. Los edificios escolares fueron construidos con las recomendaciones de las ideas higienistas como "espacios abiertos, el gimnasio y el jardín y los patios interiores aportaban condiciones de salubridad y de orden estético" (BARBIERI, 2016, p.41) era el espacio idóneo, para las actividades escolares de la escuela moderna.

El informe del presidente del CNE doctor José María Gutiérrez, respecto a la creación y construcción de edificios escolares, reseña la precariedad de las escuelas, justifica el esfuerzo presupuestal del gobierno por acondicionar o construir edificios con las condiciones mínimas para su funcionamiento; describe la carencia de espacios y materiales de los centros escolares es un símil de lo que sucedía en los países latinoamericanos, como en el caso de México.

\footnotetext{
1 Archivo digital de la Biblioteca Nacional de Maestros, Argentina (BNM). El Monitor de la Educación Común, Publicación Oficial de la Comisión Nacional de Educación, Año 1. Buenos Aires, septiembre de 1881. Núm. 1
} 


\section{$(c)$ EY}

El informe del presidente del CNE señala que en la ciudad capital, había 80 centros escolares "existiendo al mismo tiempo 55 escuelas que ocupan casas arrendadas"2 refiere que en las provincias, inició la edificación de escuelas, principalmente en Santa Fé y Corrientes; señala dos diferencias significativas: las escuelas que funcionan en casas rentadas, y las que sus edificios se encuentran en proceso de construcción; "ya cavados sus cimientos, bajo la iniciativa progresista" 3 de las autoridades educativas de las provincias. Se explica que en todo el país había 509 escuelas de instrucción primaria. Otros temas del informe fueron: la reforma a los planes de estudio bajo los preceptos de la pedagogía moderna y los libros de texto como recursos didácticos.

La capital de Argentina, en 1896 se dividía en veinte distritos escolares, distribuidos en las zonas poblacionales. Las escuelas argentinas; además de la vigilancia eran atendidas por los supervisores escolares, por un cuerpo médico escolar; que tenían como finalidad la "inspección de las escuelas públicas y particulares, visitas hechas a los domicilios de los niños afectados de enfermedades contagiosas, vacunaciones y revacunaciones practicadas, informes y certificaciones, etc." 4

El gobierno argentino inauguró el $1^{\circ}$. de junio de 1884 catorce edificios escolares según la crónica preservada en el boletín Monitor de la Educación Común ahí se describe y narra la ceremonia en donde estuvieron presente los funcionarios públicos. El presidente de la República, sólo asistió a la apertura del edificio que se localizaba "en el ángulo de las calles de Cochabamba y Entre - Ríos." ${ }^{\prime 5}$ En la ceremonia protocolaria, asistieron los cadetes de la escuela naval, de cabos y sargentos, un regimiento de infantería y un escuadrón de artillería, que hizo disparos de salva de veinte disparos al terminar la ceremonia. Los funcionarios de la Comisión Nacional de Educación, el secretario de Hacienda doctor Plaza, y el ministro de Instrucción Pública y Relaciones Exteriores, miembros del Estado Mayor del ejército acompañaron al presidente. La ceremonia representa el compromiso político del gobierno y del Estado de ofrecer educación popular a la población argentina.

\footnotetext{
${ }^{2}$ El Monitor de la Educación Común, Publicación Oficial de la Comisión Nacional de Educación, Año XVI. Buenos Aires, Marzo de 1896. Núm. 272 (1896) 530. BNM.

${ }^{3}$ El Monitor de la Educación Común, Publicación Oficial de la Comisión Nacional de Educación, Año XVI. Buenos Aires, Marzo de 1896. Núm. 272 (1896) 530. BNM.

${ }^{4}$ El Monitor de la Educación Común, Publicación Oficial de la Comisión Nacional de Educación, Año XVI. Buenos Aires, Marzo de 1896. Núm. 272. (1896) 534. BNM.

${ }^{5}$ El Monitor de la Educación Común, Publicación Oficial de la Comisión Nacional de Educación, Año. III. Buenos Aires, Junio1884. Núm. 60 (1884) 620. BNM.
} 


\section{$(c)$ EY}

La inauguración de los catorces edificios fue la respuesta del gobierno para impulsar la educación pública a la población infantil. Los discursos pedagógicos y políticos aluden a educar a la población infantil, creación de aulas y espacios adecuados a las necesidades de los alumnos.

Vamos sustituyéndolas por casas propias, construidas con arreglo a las leyes de la higiene escolar y a los adelantos de la arquitectura moderna, lo que además ofrece una gran ventaja económica como hemos sustituidos los antiguos métodos y sistemas por aquellos que la lógica y la práctica ha reconocido como buenos en Alemania, en Suiza y en Estados Unidos. ${ }^{6}$

La influencia europea y norteamericana de la educación moderna en la Argentina, quedó documentada en las fuentes historiográficas de la educación, en los discursos políticos y pedagógicos de los funcionarios de las Comisión Nacional de Educación, se alude a la necesidad de mejorar los edificios escolares, integrando áreas como la biblioteca, los museos, los salones de clase y materiales educativos de acuerdo a los ordenamientos de la pedagogía moderna.

Los edificios escolares reflejan las concepciones pedagógicas: espacio para la alfabetización y la cultura, para las actividades lúdicas, para la convivencia e interacción de los alumnos, para realizar sus necesidades fisiológicas, y lugar para la lectura. El Estado institucionaliza el espacio para que los estudiantes se formen en los ideales del ciudadano que la nación necesita dentro del contexto de la modernidad. Aunque los discursos también reconocen la precariedad en la que se encuentran la mayoría de los edificios escolares a fines del siglo XIX; arrendamiento y adaptación de casas particulares como escuelas, también las adquiría el Estado y las iba rehabilitando o construía nuevos edificios.

El análisis de los planos de 1894, ayuda a inferir que el Estado argentino implementó una política pública para la creación de edificios escolares para la población escolar. El diseño arquitectónico, demuestra que había una uniformidad en la distribución de los espacios para la tarea educativa. Los datos recabados permiten reconstruir que en el distrito $1^{\circ}$., había tres instituciones; el distrito 2do., sólo tenía dos centros escolares; distrito 3ero., contaba con cuatro escuelas; el distrito $4^{\circ}$., contaba con tres centros escolares; del $6^{\circ}$. distrito sólo se localizó una escuela; del distrito $12^{\circ}$., se identificaron dos escuelas; del distrito $14^{\circ}$., se ubicó un centro escolar; y tres edificios escolares en el distrito 15 $^{\circ}$., todos en la ciudad de Buenos Aires. Los planos de 1894

\footnotetext{
${ }^{6}$ El Monitor de la Educación Común, Publicación Oficial de la Comisión Nacional de Educación, Año. III. Buenos Aires, Junio1884. Núm. 60. (1884) 620. BNM.
} 


\section{(c) $) \mathrm{EY}$}

corresponden a 20 edificios escolares que fueron construidos para atender la educación de la población de la capital de la República Argentina.

El plano como representación cartográfica de la superficie en la que se construyeron los edificios escolares en la ciudad de Buenos Aires, tienen definidos los espacios, acotados para las actividades físicas, académicas centradas principalmente en las aulas, las salas o galería, algunas con gimnasio, laboratorio, comedores, áreas administrativas y oficinas de la dirección. Del análisis de los veinte planos de los edificios escolares se localizó, que no todas las escuelas tenían las mismas dimensiones o superficies; lo que sí se identificó es que eran edificios de dos plantas; los inmuebles fueron construidos para que acudieran niñas y niños a sus actividades escolares como lo exigía la educación moderna. El edificio y el espacio escolar construido para el aprendizaje y la enseñanza de alumnos y profesores respectivamente, estaba "destinado exclusivamente a la enseñanza, diseñado y construido con tal fin, y arquitectónicamente identificables por una serie de rasgos morfológicos, visibles y funcionales" (VIÑAO, 2008, p. 18) que la arquitectura e higiene escolar propusieron como modelo arquitectónico del edificio escolar.

En el análisis de los planos se encontró que el número de aulas varía entre cada una de las escuelas: tres edificios tenían once aulas, diez edificios contaban quince aulas, uno doce aulas, uno de diez aulas, uno de nueve aulas, uno de ocho aulas y otro de seis aulas, Las escuelas tenían entre doce y trece aulas, haciendo un promedio general de 11.7 aulas por centro escolar; lo anterior demuestra que eran centros escolares con una alta población escolar en la capital del país. Es pertinente acotar que las aulas son los espacios donde se concretizan los procesos de enseñanza y aprendizaje; de la cantidad que tenga una escuela, depende también su población escolar.

La tabla No. 1 sintetiza el número de edificios analizados y la distribución de los espacios para las tareas escolares.

TABLA 1 - Edificios Escolares en la ciudad de Buenos Aires, Argentina en el Siglo XIX.

\begin{tabular}{|c|c|c|c|c|c|c|c|c|c|c|c|c|}
\hline EDIFICIO & DISTRITO & DOMICILIO & AULAS & PATIO & $\begin{array}{c}\text { SALAS } \\
\text { GALERÍA }\end{array}$ & SANITARIOS & BODEGA & DIRECCIÓN & GIMNASIO & BIBLIOTECA & LABORATORIO & $\begin{array}{l}\text { COMEDOR } \\
\end{array}$ \\
\hline \multirow{5}{*}{$\begin{array}{l}\text { Escuelas } \\
\text { (dos } \\
\text { plantas) }\end{array}$} & \multirow{4}{*}{$\begin{array}{c}\text { Distrito } \\
1^{\circ} .\end{array}$} & $\begin{array}{c}\text { Calle } \\
\text { Esmeralda } \\
\text { No. } 287\end{array}$ & 11 & 1 & 1 & 2 & 1 & 1 & 0 & 0 & 0 & 0 \\
\hline & & $\begin{array}{c}\text { Cuyo No. } \\
790 \\
\end{array}$ & 11 & 1 & 0 & 2 & 1 & 1 & 0 & 0 & 0 & 0 \\
\hline & & $\begin{array}{c}\text { San Martín } \\
\text { No.456 }\end{array}$ & 6 & 1 & 0 & 2 & 1 & 1 & 0 & 0 & 0 & 0 \\
\hline & & $\begin{array}{l}\text { Reconquista } \\
\text { No. } 561\end{array}$ & 9 & 1 & 2 & 2 & 1 & 1 & 0 & 0 & 0 & 0 \\
\hline & & Suipacha & 11 & 1 & 2 & 2 & 1 & 1 & 0 & 0 & 0 & 0 \\
\hline
\end{tabular}

Rev. Iberoam. Patrim. Histórico-Educativo, Campinas (SP), v. 6, p. 1-21, e020009, 2020. 


\section{$(\mathrm{cc}) \mathbf{E Y}$}

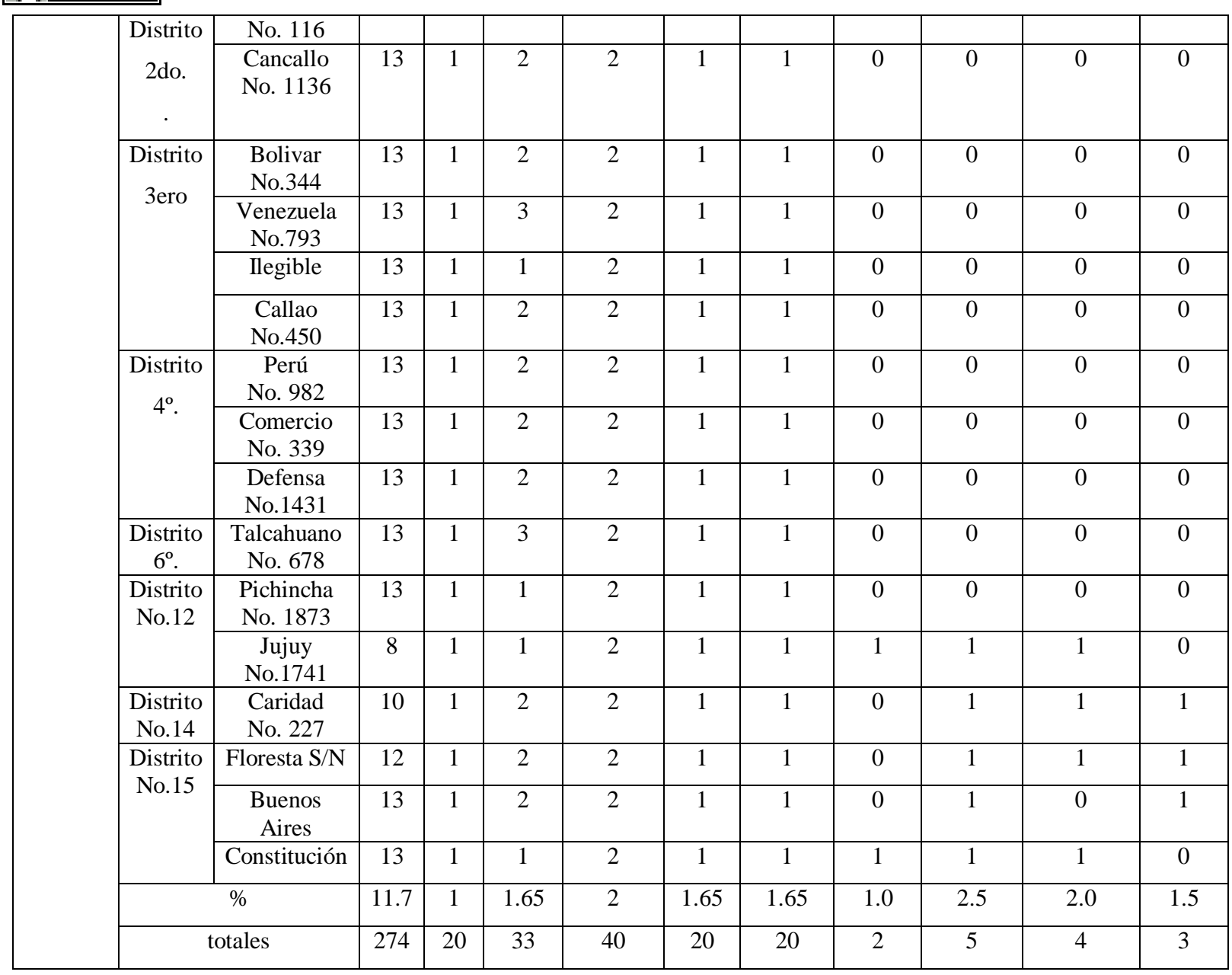

Fuente: Elaboración Propia. Datos tomados de concentrado planos de los edificios escolares del Consejo Nacional de Educación, Sala de sesiones, Edificios escolares, Capital 1894, que se localiza en la Biblioteca Nacional de Maestros en la ciudad de Buenos Aires Argentina.

El cien por ciento de los planos de los centros escolares, tenían señalado un patio para el recreo o la convivencia de los niños, niñas y maestros. Once edificios escolares contaban con dos salas o galerías. Tres escuelas tenían hasta tres salas, cinco edificios tenían sólo un espacio para sala, y dos escuelas carecían de dichos espacios; las salas o galerías servían a los alumnos y profesores para desarrollar actividades complementarias a la educación de los estudiantes.

Los edificios de las veinte escuelas de la ciudad de Buenos Aires, tienen diseñados dos módulos de sanitarios, una bodega para concentrar materiales y muebles, sólo a dos se les identificó gimnasio; cinco contaban con una biblioteca, cuatro con laboratorio y sólo a tres se les identificó comedor, lo anterior de acuerdo a la distribución en los planos de cada uno de los centros escolares. El siguiente plano ejemplifica lo referido a lo largo de este apartado. 


\section{$(\mathrm{cc}) \mathrm{EY}$}

FOTOGRAFÍA 1 - Plano de Edifico Escolar del Distrito No.1 Calle Esmeralda 257, Ciudad de Buenos Aires.

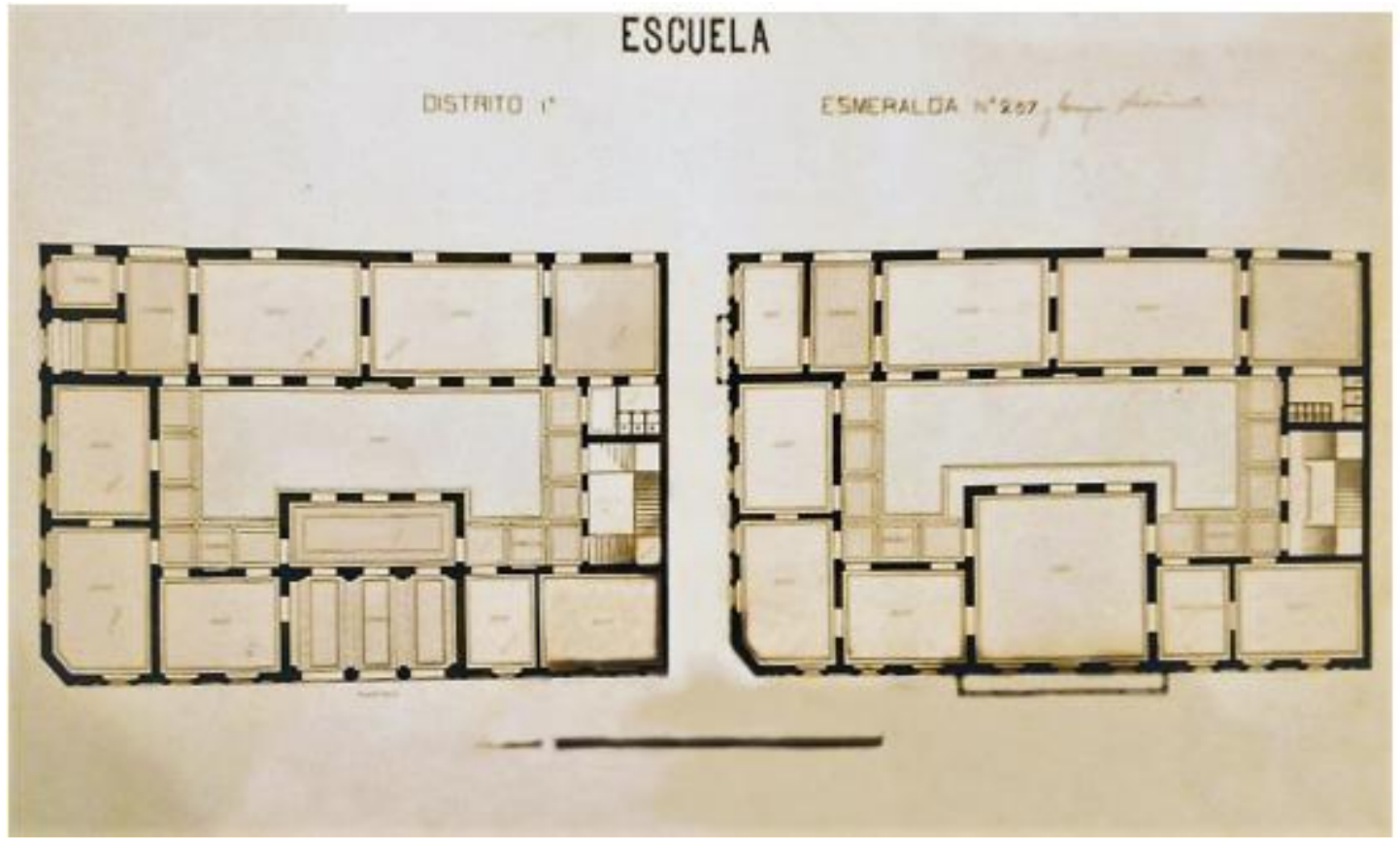

Fuente: Colección de planos de 1894 Biblioteca Nacional de Maestros. Buenos Aires, Argentina.

La construcción de edificios escolares a fines del siglo XIX en la República Argentina, refleja el interés del gobierno por proporcionar educación a la población, dentro de un modelo de educación moderna; en donde las ideas ilustradas basadas en la ciencia y la pedagogía, se traducen en acciones educativas en la formación de la ciudadanía y lograr la unidad nacional.

\section{LAS PRIMERAS INICIATIVAS EDUCATIVAS PARA LA CONSTRUCCIÓN DE LOS EDIFICIOS ESCOLARES EN MÉXICO.}

Los maestros, pedagogos, médicos, inspectores escolares, autoridades educativas y los representantes del gobierno mexicano, discuten en 1882 la necesidad de reorientar la educación de acuerdo a los preceptos del positivismo y de la educación moderna, basados en la ciencia, en las ideas liberales de democracia, justicia y libertad. Reconocen el rol del profesor y la función de la escuela de considerar que "el niño se convertiría en objeto principal de los educadores" (LOYO, 2010, p. 129). El gobierno porfirista impulsó la creación de escuelas públicas de instrucción 


\section{$(c c)$ EY}

primaria, pero no logró la cobertura del pais; si no al contrario hubo rezago, principalmente en las zonas rurales del territorio nacional.

El Congreso Higiénico-Pedagógico, fue un referente sociopolítico-educativo del Porfiriato, ahí se discutieron y acordaron acciones para el mejoramiento de las instituciones educativas, que impedían su funcionalidad como lo exigía la pedagogía y la educación moderna. Las escuelas mexicanas a fines del siglo XIX funcionaban en casas habitación adaptadas, rentadas por el Estado, con el conflicto de que los dueños no aceptaron que fueran modificadas o adaptadas. Otros condicionaban que al término del contrato, el gobierno debía dejarlas en condiciones óptimas; esto siempre provocó pérdida económica al erario público. Otras las aperturaron en locales, no aptos para las tareas educativas. Algunas escuelas funcionaban en casas de vecindad por lo que las habitaciones eran reducidas, con serias dificultades en la iluminación y ventilación.

Las conclusiones del Congreso Higiénico-Pedagógico sobre la construcción de los edificios escolares fueron las siguientes: el tipo de terreno "seco y elevado, porque el vapor de agua es el vehículo de las materias orgánicas, las cuales entran en descomposición pútrida cuando la temperatura no es muy baja, y lo segundo, para facilitar la renovación de un aire puro y saludable."7 Los materiales de construcción "menos higroscópicos, usar tezontle, ladrillo refractario, los ladrillos de arcilla vitrificada y perforados. ${ }^{8}$ El tipo de piso debía ser elevado sobre el nivel de la calle, los techos con soporte rieles y la madera de cedro. La elevación de la escuela, era una condición indispensable. Las escuelas debían estar alejadas de fábricas e industrias, porque éstas producen mal olor o problemas en el ambiente, alejada de lugares insalubres, peligroso o incomodos. Cuidar la orientación, y los vientos que favorezcan la ventilación, evitando los fríos y velocidad del viento, todo lo anterior influía en la creación de un ambiente propicio para la educación.

La construcción de los primeros edificios escolares, con las características físicas de espacio y arquitectura conforme a las recomendaciones de la pedagogía y medicina, se construyen a fines del siglo XIX. En el ocaso del Porfiriato las construcciones escolares, tomaron como base las recomendaciones del Congreso Higiénico-Pedagógico, con influencia de la arquitectura francesa en su diseño arquitectónico:

\footnotetext{
${ }^{7}$ Memorias del primer Congreso Higiénico-Pedagógico: reunido en la Ciudad de México el año de 1882 . Biblioteca Nacional de Medicina de EE. UU.

${ }^{8}$ Memorias del primer Congreso Higiénico-Pedagógico: reunido en la Ciudad de México el año de 1882. Biblioteca Nacional de Medicina de EE. UU.
} 
El modelo propuesto por el arquitecto Nicolás Mariscal suponía salones para 50 alumnos, con una superficie de $64 \mathrm{~m} 2$ y $4.50 \mathrm{~m}$ de altura. En el emplazamiento se buscó que los alumnos - sentados en pupitre- recibieran luz por el lado izquierdo y buena ventilación; condiciones de habitabilidad retomados de los tratados franceses de Louis Cloquet y Julien Guadet, además de la influencia artística de la época. (LOZADA, 2011, p. 71).

En 1905 empiezan a construirse los primeros edificios, su avance si limitó con el advenimiento de la Revolución, fueron los gobiernos posrevolucionarios quienes a partir de la década de los veinte, intensificaron la creación de edificios escolares prediseñados, con los espacios adecuados para las tareas pedagógicas en México.

La ciudad de San Luis Potosí, se localiza en la zona centro-noreste de la República Mexicana, a fines del siglo XIX en la plenitud del Porfiriato, había evolucionado significativamente en lo social, cultural y económico. El control político como característica del gobierno porfirista, también se percibía a nivel local con la autoridad de Carlos Díez Gutiérrez como uno de los gobernadores que por más de quince años administró los destinos de la entidad. Su gobierno introdujo los preceptos de la educación moderna y del positivismo en la reforma educativa de 1884.

Hubo nuevos discursos pedagógicos de cómo educar a los niños y jóvenes, de los materiales educativos, la preparación de los profesores bajo los nuevos preceptos de la educación moderna; como ejemplo a nivel local se experimentó la enseñanza objetiva "una nueva metodología para las escuelas elementales" (HERNANÁNDEZ, 2016 p.50), pero poco se transformaron los espacios educativos. El profesor Reynaldo Morales, quien fungía como segundo inspector escolar en la capital, elaboró un compendio de planos de las escuelas públicas en 1889. Su preservación en el Archivo del Sistema Educativo Estatal Regular de la entidad, permitieron elaborar este trabajo. El análisis de $\operatorname{los}_{\text {planos }}{ }^{2}$ ayudan a inferir las condiciones en las que funcionaban los edificios y espacios escolares.

El compendio contiene veinticinco planos, el diseño de cada uno, corrobora que los edificios que funcionaban como escuela, eran casa - habitación, rentadas en su mayoría, otras eran propiedad del gobierno, pero ningún edificio había sido construido exprofeso para la tarea educativa. La ciudad se dividía en siete barrios y en cada uno, había dos escuelas; una para niñas y

\footnotetext{
${ }^{9}$ AHSEER. Compendio de planos de los establecimientos públicos de la ciudad de San Luis Potosí. Elaborados en 1889 por el segundo Inspector de educación profesor Reynaldo Morales. Expediente. Dirección General. 1889.
} 


\section{$(c)$ EY}

otra para niños. Algunas escuelas tenían una sección de párvulos; antecedente de los jardines de niños.

Los edificios escolares, primero fueron casa-habitación, los veinticinco edificios tienen señalado un zaguán, con techo de viga y piso de ladrillo; fachadas del periodo colonial, con un alto grado de deterioro; en todas se localiza un patio empedrado, sólo una casa-escuela se le señala en el plano un comedor, veintidós escuelas tiene localizado un salón para las clases; éste es de dimensiones suficientes para atender a los alumnos. Sólo tres tienen dos salones para las clases; dos casa-escuela, tienen cuatro cuartos habitación sin uso, tres escuelas tienen tres cuartos sin uso, once sólo tiene los salones de clase; es decir eran los cuartos de la casa que se adaptaron, dos casasescuela tenía siete habitaciones sin uso en tareas escolares, una casa-escuela tenía ocho cuartos, una con cinco cuartos adicionales, una tenía dos añadidos y una con seis cuartos anexos. Los cuartos adicionales, al que fungía como salón de clase, eran espacios que no correspondían al diseño arquitectónico de una escuela, demuestran que eran casas adaptadas para las actividades escolares. Se identificó en una escuela que tenía ubicado en el plano una caballeriza.

Los planos, tienen definida una sección de párvulos, en doce edificios, se señala espacio de cocina, en siete instituciones se identifica en el plano que tenían cuartos sin techo, porque estos habían colapsado por poco mantenimiento para su preservación. Dos centros escolares contaban con bodegas para guardar bastidores, aparatos y materiales para gimnasia. Los veinticinco centros escolares contaban con una sección de sanitarios. La mitad de las escuelas contaba con pozo para sustraer el agua para el consumo de los alumnos de cada escuela. Del plano de la escuela No.8 ejemplifica la casa habitación adaptada como espacio escolar. 


\section{$(\mathrm{cc}) \mathrm{EY}$}

FOTOGRAFÍA 2 - Plano de la Escuela de Instrucción Elemental No.8

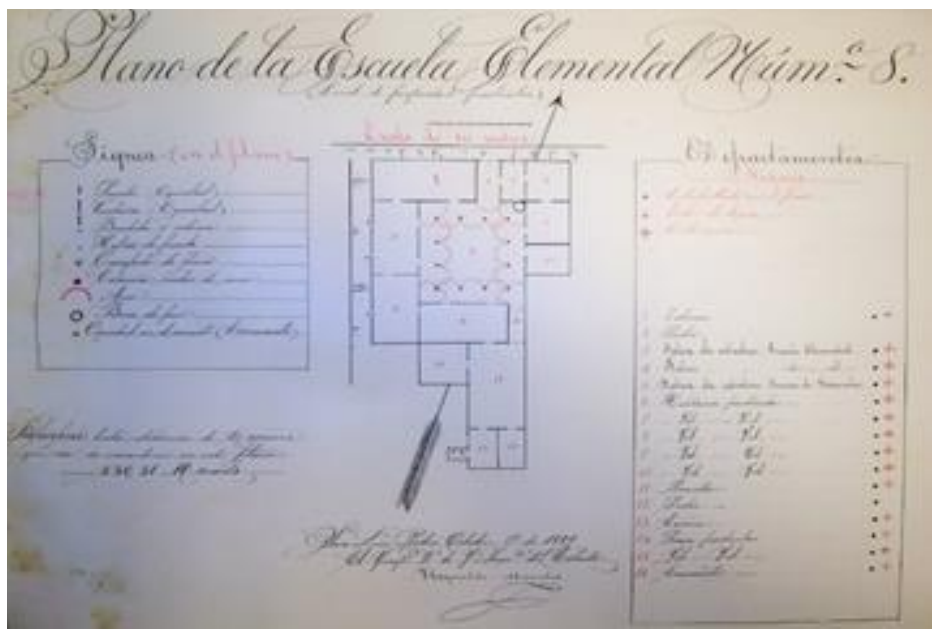

Fuente: Archivo Histórico SEER.México. Compendio de planos de 1889 de las escuelas públicas de la ciudad de San Luis Potosí

Los datos recabados de los planos de las escuelas de instrucción primaria en San Luis Potosí, demuestran que, aunque en los discursos se menciona la importancia y necesidad de crear espacios adecuados para la tarea educativa de acuerdo a las exigencias de la educación moderna; la infraestructura física de los centros educativos, no ayudaba a que se concretizaran los ideales de la educación moderna. Caso contrario sucedía en Argentina, ahí el gobierno había creado edificios para la tarea educativa, por tanto, estaban en mejores condiciones, la atención de los estudiantes, con mayor apego a los preceptos de la educación moderna del siglo XIX, principalmente en la ciudad de Buenos Aires.

La primera escuela construida por el gobierno del estado de San Luis Potosí, fue hasta el siglo XX, cuya inauguración fue en el año de 1907.

El edificio pertenece al movimiento arquitectónico ecléctico que imperaba todavía a principios del siglo en la ciudad, aunque se inclinaba hacia el neoclásico. $\mathrm{Su}$ fachada está construida con piedra gris (extraída de la cantera de los cortes de la hacienda de Bocas), y ladrillo prensado de color rojo y amarillo. Es un edificio de una sola planta, elevado unos noventa centímetros sobre el nivel de la acera. (VILLAR, 2000, p. 150).

Al hacer un análisis de los planos de la primera escuela modelo se localizan los siguientes espacios, que de acuerdo a los requerimientos de la escuela moderna y de la corriente de higiene escolar que en Europa y los Estados Unidos, ya se habían institucionalizado desde fines del siglo 


\section{$(c)$ EY}

XIX, en San Luis Potosí, este primer edificio, marcó una ruptura e inaugura una nueva etapa de la educación pública en la entidad. La imagen del plano No. 3 demuestra la distribución de los espacios del primer edificio escolar.

FOTOGRAFÍA 3 - Plano del primer edificio escolar elabarorado por el Ing. Encarnación Ipiña en la Ciudad de San Luis Potosí.México.

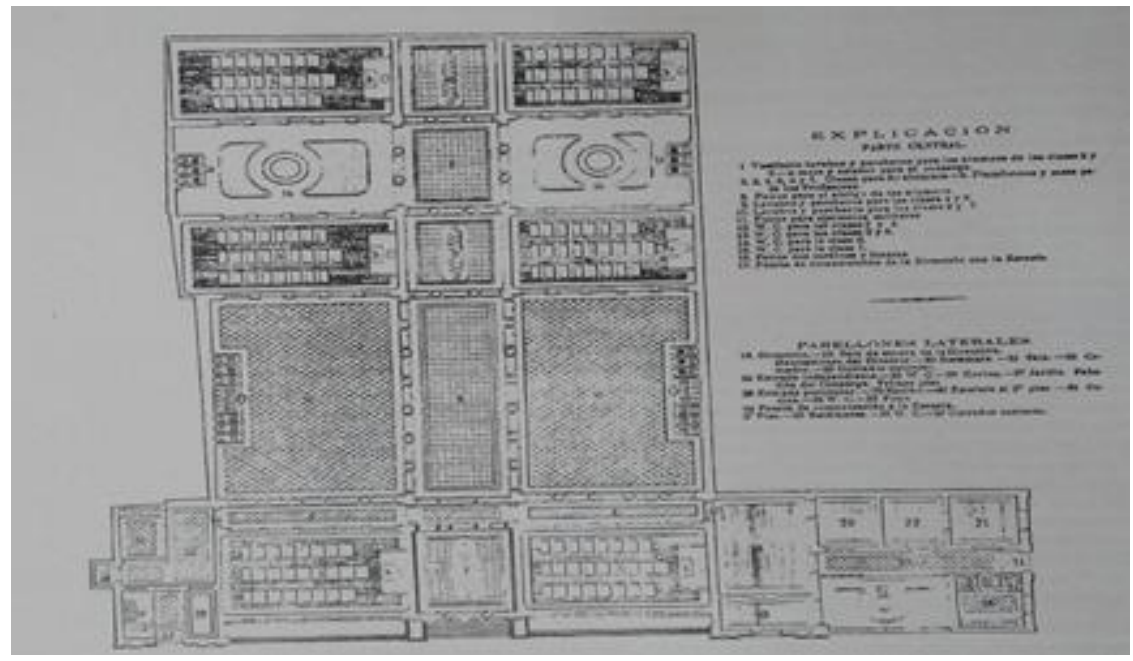

Fuente: Plano tomado de la obra VILLAR Rubio José Victoriano. El centro histórico de la ciudad de San Luis Potosí. San Luis Potosí-México. Universidad Autónoma de San Luis Potosí: Editorial Universitaria Potosina, 2000.

Los planos ubican una parte central del edificio; contiene vestíbulo, lavabos y percheros en cada sección para los alumnos de las clases, mesas y asiento para el conserje, seis aulas con capacidad para atender a cincuenta alumnos, cada aula con su plataforma y mesa para profesores. Patios pavimentados con mosaico para el abrigo de los alumnos, otros patios para ejercicio militares, patios con jardines y fuentes. Cuatro módulos de sanitarios para los estudiantes.

Los planos de los pabellones laterales, tienen la dirección, sala de espera de la dirección, habitaciones del director, recámaras, salas, comedores y corredor cubierto, con entradas independientes, con su sanitario, cocina, jardín, pabellón de conserje, correspondiente, primer piso. Por primera vez en San Luis Potosí, se creó el primer edificio construido especialmente para atender las actividades escolares, con espacios diseñados de acuerdo a las aportaciones científicas de la higiene, arquitectura, ingeniería y pedagogía. La construcción de edificios escolares se convirtió en una política educativa del Estado Mexicano, que se intensificó durante el siglo XX 


\section{$(\mathrm{cc}) \mathbf{E Y}$}

después de la Revolución Mexicana, al considerarse a la educación como un derecho social bajo la responsabilidad del Estado.

\section{ANÁLISIS Y COMENTARIOS FINALES}

Los edificios escolares de las ciudades de Buenos Aires en Argentina y de San Luis Potosí en México respectivamente, reflejan el grado de evolución de los sistemas educativos de estos dos países. Comprender la complejidad en la que se da la creación de los primeros edificios escolares, como espacios para la enseñanza, los datos antes analizados, permiten inferir el rol del Estado como garante del derecho a la educación pública o popular; las decisiones políticas que tomó el Estado a fines del siglo XIX para crear escuelas, profesores, invertir en materiales educativos y mobiliario es evidencia de su interés por introducir las ideas de la modernidad en la educación nacional. La educación como medio para la consolidación de las ideas liberales del Estado; adquiere características ideologizantes desde los centros escolares. La República de Argentina y la República Mexicana, a través de los ministerios de Justicia, Culto e Instrucción Pública y Justicia e Instrucción y Bellas Artes, respectivamente, definían en razón a las necesidades sociopolíticas de la época, cómo debía ser la educación.

Cada país tiene procesos diferenciados en el desarrollo educativo a fines el siglo XIX; aunque en ambos países la influencia del liberalismo y positivismo se refleja en la educación, y en las decisiones políticas para generar las condiciones óptimas para que la educación popular o pública cumpla su función formativa e ideológica de cada ciudadano que educa el Estado.

La construcción de edificios escolares exprofeso, es un ejemplo de las ideas de renovación pedagógica de la modernidad. Argentina a fines del siglo XIX, en la ciudad de Buenos Aires, integra al sistema educativo las ideas de la educación moderna. Las ideas ilustradas, del papel de la ciencia, sólo puede concretizar, si existen espacios educativos que satisfagan los requerimientos de la pedagogía. A fines del siglo XIX se están construyendo edificios con aulas, salas, galerías, gimnasio, comedores y laboratorios. El espacio escolar, es lugar del estudio de la ciencia, también de convivencia, de la recreación y la cultura. Las nuevas construcciones escolares se justifican desde los discursos políticos y pedagógicos, como sinónimo de la modernidad.

Los postulados de la educación moderna se diseminaron en los congresos de instrucción primaria, en las escuelas normales y por pedagogos como Enrique C. Rébsamen, Justo Sierra, 


\section{$(c)$ EY}

Joaquín Baranda y Gregorio Torres Quintero. Los pedagogos, maestros y médicos discutieron la necesidad de salir de la instrucción centrada solamente en el conocimiento, y la urgente necesidad de pasar a una concepción integral del desarrollo de los niños y jóvenes en lo cognitivo, psicomotor y socio-afectivo. Además de crear los espacios específicos para la enseñanza y el aprendizaje, concretamente los edificios escolares. El Congreso Higiénico-Pedagógico, fue donde confluyeron las ideas de la pedagogía moderna, la medicina y la higiene como disciplinas que aportaban conocimiento para transformar la educación. Las conclusiones a las que llegaron los asistentes del congreso: mejorar los métodos de enseñanza y materiales educativos; precariedad de los edificios de las escuelas mexicanas; la urgente necesidad de que el país avance hacia la modernidad; asimetría en la desarrollo urbano y rural de las ciudades y comunidades del Porfiriato.

Tanto en la ciudad de Buenos Aires, como en la ciudad de San Luis Potosí, las comisiones encargadas de administrar la educación la componían profesores, pedagogos y médicos. Por primera vez en la historia de la pedagogía se debatía la importancia de la construcción de los centros educativos por el Estado, pero sobre todo la aportación interdisciplinaria de ingenieros, médicos, maestros y pedagogos al correlacionar el conocimiento de sus disciplinas para crear edificios y espacios simbólicos para el estudio y trabajo escolar.

La creación de los edificios escolares como espacios simbólicos de la modernidad, sintetizan los avances de la ciencia y las disciplinas: pedagogía, medicina, ingeniería y arquitectura. El espacio escolar, fue concebido como lugar común creado por el Estado, al que acuden los estudiantes para apropiarse de la cultura y de la ideología que subyace a la acción educativa. Los edificios escolares que se construyeron a fines del siglo XIX en la ciudad de Buenos Aires, como lo testimonian los planos de 1894 eran construcciones que representaban la aspiración educativa del Estado enmarcado dentro del contexto de la modernidad.

En México, en la ciudad de San Luis Potosí, los planos de 1889 reflejan las condiciones de precariedad en la que se encontraban las escuelas públicas -no había edificios exprofeso-. Los espacios escolares fueron creados en casas habitación, en casas rentadas o comparadas por el gobierno, pero no cumplían con las exigencias de la Modernidad, que, en Europa, ya existían, y que, en México, los primeros edificios escolares aparecieron hasta la primera década del siglo XX.

Las construcciones de los edificios escolares y sus espacios reflejan el poder del Estado, el ideal de educación y ciudadano que se forman a través de la educación. Las diferencias entre la ciudad de Buenos Aires y la ciudad de San Luis Potosí en México, es resultado de la evolución 


\section{$(\mathrm{cc})$ EY}

histórica y de las condiciones del contexto sociopolítico e histórico de cada país. Las similitudes que se identifican en estas dos latitudes, es el interés del Estado, por ofrecer una educación que forme al sujeto para integrarse a la vida social y política, como ciudadano, bajo los preceptos de la ciencia, y la moralidad que el Estado considera pertinente para la convivencia social: esto es incuestionable en el interés de cada uno de los países.

\section{ARCHIVOS}

Archivo del Sistema Educativo Estatal Regular, SLP. México.

Biblioteca Nacional de Maestros, Argentina.

Biblioteca Nacional de Medicina de EE. UU.

\section{BIBLIOGRAFÍA}

BARBIERI, Patricia. Arquitectura para la educación e higienismo: el Hospital e Instituto de Enseñanza Médica del Centenario y la Escuela Normal n. ${ }^{\circ}$ «Dr. Nicolás Avellaneda» en la ciudad de Rosario. Revista de Historia de la educación, v.17, n.2, p. 31-52, 2016.

BAZANT, Mílada. "Los Congresos de Instrucción y sus principios rectores", en Historia de la educación durante el porfiriato. México, El Colegio de México, pp. 21-30, 1995.

CHAOUL, María. La higiene escolar en la Ciudad de México en los inicios del siglo XX. Historia Mexicana, Editorial El Colegio de México, A.C., v. LXII, n. 1, p. 249-304, 2012.

GODÍNEZ Visedo José Miguel. Espacio escolar y reforma de la enseñanza. Revista interuniversitaria de formación del profesorado, 11, p. 125-135, 1991.

GUTIÉRREZ, María Teresa. Proceso de institucionalización de la higiene: estado, salubridad e higienismo en Colombia en la primera mitad del siglo XX. Estudios Socio-Jurídicos, [S.l.], Colombia, v. 12, p. 73-97, 2010.

HERNÁNDEZ Ortiz, Francisco. Compendio de planos de los Establecimientos Públicos de San Luis Potosí a finales del siglo XIX. México: Editorial Pedro Vallejo, 2011.

HERNÁNDEZ Ortiz, Francisco. La Pedagogía eje de la formación del profesorado a fines del siglo XIX en El patrimonio Histórico Educativo: el acervo y los libros de higiene escolar, pedagogía, economía doméstica y geografía en la formación del profesorado. México: Ediciones del Lirio, 2016.

LAHOZ Abad, Purificación. El modelo froebeliano de espacio-escuela. Su introducción en España. Historia de la Educación, Salamanca, v. 10, p. 107-133, 2010. 


\section{(c) $) \mathrm{EY}$}

LIONETTI, Lucía. Discursos, Representaciones Y Prácticas Educativas sobre el Cuerpo de los Escolares: Argentina en las primeras Décadas Del Siglo XX. Cuadernos de Historia, Santiago, p. 31-52, 2011.

LIONETTI, Lucía. La función republicana de la escuela pública. La formación del ciudadano en Argentina a fines del siglo XIX. Revista Mexicana de Investigación Educativa, 10(27), p. 1225$1255,2005$.

LOYO, Engracia, Anne Staples. Fin del siglo y de un régimen en La Educación en México. México: El Colegio de México, 2010.

LOZADA, Santa Ana Lucia. Arquitectura escolar en México. Bitácora arquitectura, [S.l.], n. 17, p. 70-75, 2011.

MALDONADO Tapias, et al. Historia de la arquitectura escolar en Colombia. Universidad Nacional de Colombia-Unibiblos, 1999.

MARENGO, Roberto. Estructuración y consolidación del poder normalizador: el Consejo Nacional de Educación en Puiggrós, A., Puiggrós, A., \& Carli, S. Sociedad civil y Estado en los orígenes del sistema educativo argentino. Argentina: Editorial Galerna. (Vol. 2), 1991.

MEMORIAS del primer Congreso Higiénico-Pedagógico: reunido en la Ciudad de México el año de 1882. México: Imprenta del Gobierno en Palacio.1883. Open Knowledge Commons, Biblioteca Nacional de Medicina de EE. UU. Disponible: https://archive.org/details/23911420R.nlm.nih.gov

PINEAU Pablo, DUSSEL Inés y CARUSO, Marcelo. La escuela como máquina de educar. Buenos Aires: Editorial Paidós, 2001.

RODRÍGUEZ Méndez Francisco Javier. Arquitectura escolar en España: 1857-1936. Tesis de doctorado en Universidad Politécnica de Madrid. Arquitectura, p. 1-897, 2004.

VILLAR Rubio José Victoriano. El centro histórico de la ciudad de San Luis Potosí. San Luis Potosí-México. Universidad Autónoma de San Luis Potosí: Editorial Universitaria Potosina, 2000.

VIÑAO Frago Antonio. Escolarización, edificios y espacios escolares. Revista Participación Educativa, 7, p. 16-27, 2008.

VIÑAO Frago Antonio. Del espacio escolar y la escuela como lugar: propuestas y cuestiones. Historia de la Educación, Revista Interuniversitaria, Madrid, 12, p. 17-74, 1993.

Recebido em: 07 de janeiro de 2020 Aceito em: 19 de junho de 2020 